Les ANNALES POLONICI MATHEMATICI constituent une continuation des ANNALES DE LA SOCIETE POLONAISE DE MATHEMATIQUE (vol. I-XXV) fondées on 1921 par Stanisław Zaremba.

Les ANNALES POLONICI MATHEMATICI publient, en langues des congrès internationaux, des travaux consacrés à l'Analyse Mathématique, la Géométrie et la Théorie des Nombres. Chaque volume parait en 3 fascicules.

Les manuscrits dactylographiés sont à expédier à l'adresse: Rédaction des ANNALES POLONICI MATHEMATICI KRAKOW (Pologne), ul. Solskiego 30.

Toute la correspondance concernant l'échange et l'administration est . à expédier à l'adresse:

ANNALES POLONICI MATHEMATICI

WARSZAWA 10 (Pologne), ul. Sniadeckich 8.

Le prix de ce fascicule est $2 \$$.

Les ANNALES sont à obtenir par l'intermédiaire de ARS POLONA

WARSZAWA (Pologne), Krakowskie Przedmieście 7.

PRINTED IN POLAND

\section{Some properties of convex functions of higher orders $\left(^{*}\right)$}

\author{
by Z. CIestelskr (Poznań)
}

In this paper we study conditions sufficient for the continuity of a convex function of the $n$-th order.

The author is very grateful to Professor W. Orlicz for a number of suggestions.

First we shall recall the definitions of a convex function of the $n$-th order in the sense of Jensen and in the sense of Popoviciu. Furthermore we establish some lemmas necessary to prove the fundamental theorems.

In the present paper we shall denote by $(a, b)$ an open interval. Let $\Delta_{h}^{n} f(x)$ denote a finite difference of the function $f$ of the $n$-th order with increment $h$ defined as follows:

$$
\Delta_{h}^{0} f(x)=f(x), \quad \Delta_{h}^{n} f(x)=\Delta_{h}^{n-1} f(x+h)-\Delta_{h}^{n-1} f(x) .
$$

Let $\left[x_{1}, \ldots, x_{n+1} ; f\right]$ denote a divided difference (diférence divisée, see [4]) of the function $f$ at the different points $x_{1}, \ldots, x_{n+1}$. This difference is determined by

$$
[x ; f]=f(x), \quad\left[x_{1}, \ldots, x_{n+1} ; f\right]=\frac{\left[x_{2}, \ldots, x_{n+1} ; f\right]-\left[x_{1}, \ldots, x_{n} ; f\right]}{x_{n+1}-x_{1}} .
$$

It is well known that

where

$$
\left[x_{1}, \ldots, x_{n+1} ; f\right]=\frac{U\left(x_{1}, \ldots, x_{n+1} ; f\right)}{V\left(x_{1}, \ldots, x_{n+1}\right)}
$$

$$
U\left(x_{1}, \ldots, x_{n+1} ; f\right)=\left|\begin{array}{ccccc}
1 & x_{1} & \ldots & x_{1}^{n-1} & f\left(x_{1}\right) \\
\ldots & \ldots & \ldots & \ldots & \ldots \\
1 & x_{n+1} & \ldots & x_{n+1}^{n-1} & f\left(x_{n+1}\right)
\end{array}\right|,
$$

$$
V\left(x_{1}, \ldots, x_{n+1}\right)=U\left(x_{1}, \ldots, x_{n+1} ; x^{n}\right)=\left|\begin{array}{cccc}
1 & x_{1} & \ldots & x_{1}^{n} \\
\cdots & \ldots & \ldots & \ldots \\
1 & x_{n+1} & \ldots & x_{n+1}^{n}
\end{array}\right| .
$$

(*) This paper constitutes a part of the paper which obtained the first place at a competition for the best mathematical student publication organized in May 1957 at the Second Polish Symposium of Scientific Mathematical Student Society in Torun. 
A function $f$ defined on $(a, b)$ will be called J-convex of the $n$-th order (in the sense of Jensen) over $(a, b)$ if the inequality

$$
\Delta_{h}^{n+1} f(x) \geqslant 0
$$

holds for all $x$ and $h>0$ such that $a<x<x+(n+1) h<b$. A function $f$ defined on $(a, b)$ will be called $\mathrm{P}$-convex of the $n$-th order (in the sense of Popoviciu) over $(a, b)$ if the inequality

$$
\left[x_{1}, \ldots, x_{n+2} ; f\right] \geqslant 0
$$

holds for all points $x_{1}, \ldots, x_{n+2}$ such that $a<x_{1}<\ldots<x_{n+2}<b$.

The fact that the definitions are equivalent for $f$ continuous on $(a, b)$ was proved earlier by T. Popoviciu in [4]. This result follows immediately from the remark that $[x, x+h, \ldots, x+(n+1) h ; f](n+1) ! h^{n+1}=$ $=\Delta_{h}^{n+1} f(x)$ and from the following

LEMMA 1 ([4]). If a function $f$ is $\mathrm{J}$-convex of the $n$-th order over $(a, b)$, then the inequality

$$
\left[x_{1}, \ldots, x_{n+2} ; f\right] \geqslant 0
$$

holds for $a<x_{1}<\ldots<x_{n+2}<b$, where the points $x_{2}, \ldots, x_{n+1}$ divide rationally the interval $\left(x_{1}, x_{n+2}\right)$.

It may be instructive to remark at this point that if a function $f$ is P-convex of the $n$-th order over $(a, b)$, it is also continuous in that interval. This follows from (1), (2) and (3) (see [4]).

Now we shall prove

LEMM' 2. Let $f$ denote a function such that for every $x_{0} \epsilon(a, b)$ and for every $\varepsilon>0$ there exist a set of measure zero (of the first category) $N=N\left(x_{0}, \varepsilon\right)$ and a number $\delta=\delta\left(x_{0}, \varepsilon\right)>0$ such that $x \epsilon\left(x_{0}-\delta, x_{0}+\delta\right)-N$ implies $\left|f(x)-f\left(x_{0}\right)\right|<\varepsilon$. Then $f$ is continuous at each point $x \in(a, b)$.

Proof. Let us suppose that $f$ is discontinuous at $x_{0} \epsilon(a, b)$. Then there exists $\varepsilon_{0}>0$ such that the oscillation $\omega\left(x_{0}\right)$ of $f$ at $x_{0}$ satisfies the condition $\omega\left(x_{0}\right)>2 \varepsilon_{0}$. By our hypothesis there exist $N_{1}=N_{1}\left(x_{0}, \varepsilon_{0} / 2\right)$ and $\delta_{1}=\delta\left(x_{0}, \varepsilon_{0} / 2\right)$ such that $x \epsilon\left(x_{0}-\delta_{1}, x_{0}+\delta_{1}\right)-N_{1}$ implies $\mid f(x)-$ $-f\left(x_{0}\right) \mid<\varepsilon_{0} / 2$. It is easily seen that a point $y_{0} \epsilon\left(x_{0}-\delta_{1}, x_{0}+\delta_{1}\right)$ may be found such that $\left|f\left(x_{0}\right)-f\left(y_{0}\right)\right|>\varepsilon_{0}$. By our assumption we find again that for certain $N_{2}=N_{2}\left(y_{0}, \varepsilon_{0} / 2\right)$ and $\delta_{2}=\delta_{2}\left(y_{0}, \varepsilon_{0} / 2\right)$ the relation $x \epsilon\left(y_{0}-\delta_{2}, y_{0}+\delta_{2}\right)-N_{2}$ implies $\left|f(x)-f\left(y_{0}\right)\right|<\varepsilon_{0} / 2$. Moreover, we impose $\left(y_{0}-\delta_{2}, y_{0}+\delta_{2}\right) \subset\left(x_{0}-\delta_{1}, x_{0}+\delta_{1}\right)$. Then $x \in\left(y_{0}-\delta_{2}, y_{0}+\delta_{2}\right)-\left(N_{1} \cup N_{2}\right)$ implies $\varepsilon_{0}<\left|f\left(x_{0}\right)-f\left(y_{0}\right)\right| \leqslant\left|f(x)-f\left(x_{0}\right)\right|+\left|f(x)-f\left(y_{0}\right)\right|<\varepsilon_{0}$. Thus $f$ is continuous on $(a, b)$.
LEMMA 3. Let $E \subset(a, b)$ denote a set of positive measure (of the second category and satisfying the condition of Baire) and $R$ the set of all rational numbers. Then $m\left[(a, b)-E_{0}\right]=0\left(E_{0}\right.$ is residual in $\left.(a, b)\right)$, where

and

$$
E_{0}=\bigcup_{r \in R} E_{r}
$$

$$
E_{\mu}=\left\{x: x=(1-\mu) x_{0}+\mu y, y \in E\right\}
$$

for any real $\mu \neq 0$ and $a$ fixed $x_{0} \epsilon(a, b)$.

Proof. We suppose that the set is measurable and of positive measure. Let $y_{0} \epsilon(a, b)$ denote any point of density of $E$. We introduce the symbols $I(\delta)=\left(y_{0}-\delta, y_{0}+\delta\right)$ where $\delta>0$ and $I_{\mu}(\delta)=\left\{x: x=(1-\mu) x_{0}+\right.$ $+\mu y, y \in I(\delta)\}$. For every $\varepsilon>0$ there exists $\delta_{0}>0$ such that $0<h<\delta_{0}$ implies $m E \cap I(h)>(1-\varepsilon) m I(h)$. We remark that for $r \in R, r \neq 0$ and $\mu \neq 0$ the inequalities

$$
\frac{m E_{0} \frown I_{\mu}(h)}{m I_{\mu}(h)} \geqslant \frac{m E_{r} \frown I_{\mu}(h)}{m I_{\mu}(h)}, \quad \frac{m E_{\mu} \frown I_{\mu}(h)}{m I_{\mu}(h)}=\frac{m E \frown I(h)}{m I(h)}>1-\varepsilon
$$

hold for $0<h<\delta_{0}$. Let $z$ denote any point of $(a, b)$ and $\lambda \neq 0$ the real number satisfying the condition $z=(1-\lambda) x_{0}+\lambda y_{0}$. Since for each $\eta>0$ and $h \epsilon\left(0, \delta_{0}\right)$ there exists $r \in R$ such that

$$
\left|\frac{m E_{r} \cap I_{\lambda}(h)}{m I_{\lambda}(h)}-\frac{m E_{\lambda} \cap I_{\lambda}(h)}{m I_{\lambda}(h)}\right|<\eta,
$$

then $0<h<\delta_{0}$ implies $m E_{0} \cap I_{\lambda}(h)>(1-\varepsilon) m I_{\lambda}(h)$. It follows at once that $m\left[(a, b)-E_{0}\right]=0$.

If $E$ is of the second category and satisfies the condition of Baire then the proof is analogous to that given above. It is sufficient to replace the points of density by the points of $E$ which are of the first category in the set $(a, b)-E$.

THEOREM' 1. 'Let the J-convex function $f$ defined over' $(a, b)$, of the $n$-th order, be bounded on a set $E \subset(a, b)$ of positive measure. Then $f$ is continuous on $(a, b)$.

Proof. Let $x_{0}$ denote any fixed point of $(a, b)$ such that $m\left(a, x_{0}\right) \frown$ $\cap E>0$. Then there exist $I_{i}=\left(x_{i 0}-\delta, x_{i 0}+\delta\right)$ and $\delta>0$ such that $a<x_{10}-\delta<x_{10}+\delta<x_{20}-\delta<\ldots<x_{n 0}+\delta<x_{0}$ where $x_{i_{0}}$ denotes the point of density of $\left(a, x_{0}\right) \cap E$ for $i=1,2, \ldots, n$. L et

$$
E_{r i}=\left\{x: x=(1-r) x_{0}+r y, y \in I_{i} \cap E\right\}
$$


and let $R$ denote the set of all rational numbers. By lemma 3 we obtain

$$
m\left[(a, b)-E_{0}\right]=0 \quad \text { where } \quad E_{0}=\bigcap_{i=1}^{n} \bigcup_{r \in R} E_{r i} .
$$

Let $\delta_{0}>0$ satisfy the condition $x_{n 0}+\delta<x_{0}-\delta_{0}<x_{0}+\delta_{0}<b$. It is easy to remark that the set $E_{0}$ has the following properties: for every $x \in I_{0} \cap E_{0}$ where $I_{0}=\left(x_{0}-\delta_{0}, x_{0}+\delta_{0}\right)$ there exist points $x_{i}$ belonging to $I_{i} \cap E$, for $i=1,2, \ldots, n$, such that the points $x_{2}, \ldots, x_{n}, x_{0}$ divide rationally the interval $\left(x_{1}, x\right)$. Since $f$ is a $J$-convex function of the $n$-th order over $(a, b)$, we have by lemma 1

$$
\left[x_{1}, \ldots, x_{n+2} ; f\right] \geqslant 0
$$

for $a<x_{1}<\ldots<x_{n_{+2}}<b$ such that $x_{2}, \ldots, x_{n+1}$ divide rationally the interval $\left(x_{1}, x_{n+2}\right)$. Inequality (6) together with (1) and. (3) implies

whence by (2)

$$
U\left(x_{1}, \ldots, x_{n+2} ; f\right) \geqslant 0
$$

(8) $f\left(x_{n+2}\right) \nabla\left(x_{1}, \ldots, x_{n+1}\right)-f\left(x_{n+1}\right) \nabla\left(x_{1}, \ldots, x_{n}, x_{n+2}\right)$

$$
\geqslant-\sum_{i=1}^{n}(-1)^{n-i} f\left(x_{i}\right) V\left(x_{1}, \ldots, x_{i-1}, x_{i+1}, \ldots, x_{n+2}\right) .
$$

Let $x_{n+1}=x_{0}$ and $x_{n+2}=x$. By (8) we obtain

(9) $f(x)-f\left(x_{0}\right) \geqslant-f\left(x_{0}\right)\left[1-\frac{V\left(x_{1}, \ldots, x_{n}, x\right)}{V\left(x_{1}, \ldots, x_{n}, x_{0}\right)}\right]$

$$
-\left(x-x_{0}\right) \sum_{i=1}^{n}(-1)^{n-i} \frac{f\left(x_{i}\right)}{x-x_{0}} \cdot \frac{V\left(x_{1}, \ldots, x_{i-1}, x_{i+1}, \ldots, x_{0}, x\right)}{V\left(x_{1}, \ldots, x_{n}, x_{0}\right)} .
$$

The intervals $I_{i}, i=1,2, \ldots, n$, are fixed and no two of them have common points. Therefore there exists a positive number $\eta>0$ such that $\left|V\left(x_{1}, \ldots, x_{n}, x_{0}\right)\right|>\eta$ for all $x_{1}, \ldots, x_{n}\left(x_{i} \in I_{i} \cap E, i=1,2, \ldots, n\right)$. Moreover we remark that:

$1^{\text {o The values }} f\left(x_{i}\right)$ do not exceed the upper bound of $f$ on the set $E$.

$2^{\circ}$ since $V\left(x_{1}, \ldots, x_{i-1}, x_{i+1}, \ldots, x_{0}, x\right)$ contains $\left(x-x_{0}\right)$ as a factor, we infer that $V\left(x_{1}, \ldots, x_{i-1}, x_{i+1}, \ldots, x_{0}, x\right) /\left(x-x_{0}\right)$ is bounded for $x_{j} \in I_{j} \cap E(j=1,2, \ldots, n)$ and $x \in\left(x_{0}, x_{0}+\delta_{0}\right)$.

Thus for $x \rightarrow x_{0}\left(x_{0}<x<x_{0}+\delta_{0} ; x \in E_{0}\right)$ the right hand side of (9) converges to zero uniformly in $x_{1}, \ldots, x_{n}$. Therefore for every $\varepsilon>0$ there exists $\delta_{1}>0$ such that

$$
x \epsilon\left(x_{0}, x_{0}+\delta_{1}\right) \cap E_{0} \quad \text { implies } \quad f(x)-f\left(x_{0}\right)>-\varepsilon .
$$

We take in (8) $x_{n+1}=x$ and $x_{n+2}=x_{0}$. Then by a method substantially the same as that we have used above we obtain the existence of $\delta_{2}>0$ such that

$$
x \in\left(x_{0}-\delta_{2}, x_{0}\right) \cap E_{0} \quad \text { implies } f(x)-f\left(x_{0}\right)<\varepsilon .
$$

Relations (10) and (11) imply the boundedness of the function $f$ below on $\left(x_{0}, x_{0}+\delta_{1}\right) \cap E_{0}$ and above on $\left(x_{0}-\delta_{2}, x_{0}\right) \frown E_{0}$. The same result will appear if we replace $x_{0}$ by $x_{0}^{\prime} \epsilon\left(x_{0}, x_{0}+\delta_{1}\right)$. Define the sets $E_{r i}^{\prime}$ and $E_{0}^{\prime}$ as in (4) and (5) using $x_{0}^{\prime}$ instead of $x_{0}$. Thus we find that there exists $\delta_{3}>0$ such that for $x \epsilon\left(x_{0}^{\prime}-\delta_{3}, x_{0}^{\prime}\right) \cap E_{0}^{\prime}$ we have $f(x)-f\left(x_{0}^{\prime}\right)<\varepsilon$, where $\left(x_{0}^{\prime}-\delta_{3}, x_{0}^{\prime}\right) \subset\left(x_{0}, x_{0}+\delta_{1}\right)$ and $x_{0}<x_{0}^{\prime}-\delta_{3}$. It follows that the function $f$ is bounded on the set $E_{1}=\left(x_{0}^{\prime}-\delta_{3}, x_{0}^{\prime}\right) \cap E_{0}^{\prime} \cap E_{0}$. Of course $m_{1}>0$. If we replace in (4) the set $I_{n} \cap E$ by $E_{1}$ then we obtain by (5) a new set $E_{0}^{\prime \prime}$ satisfying the condition $m\left[(a, b)-E_{0}^{\prime \prime}\right]=0$. We put in $(7) x_{n}=x_{0}$ and $x_{n+1}=x$. Let $x_{n+2} \in E_{1}$ and $x_{i} \in I_{i} \cap E$ for $i=1,2, \ldots, n-1$. Then $U\left(x_{1}, \ldots, x_{n-1}, x_{0}, x, x_{n+2}\right) \geqslant 0$, whence analogously to (9)

$$
\begin{aligned}
f(x) & -f\left(x_{0}\right) \leqslant-f\left(x_{0}\right)\left[1-\frac{V\left(x_{1}, \ldots, x_{n-1}, x, x_{n+2}\right)}{V\left(x_{1}, \ldots, x_{n-1}, x_{0}, x_{n+2}\right)}\right]+ \\
& +f\left(x_{n+2}\right) \frac{V\left(x_{1}, \ldots, x_{n-1}, x_{0}, x\right)}{V\left(x_{1}, \ldots, x_{n-1}, x_{0}, x_{n+2}\right)}+ \\
& +\sum_{i=1}^{n}(-1)^{n-i} f\left(x_{i}\right) \frac{V\left(x_{1}, \ldots, x_{i-1}, x_{i+1}, \ldots, x_{n-1}, x_{0}, x, x_{n+2}\right)}{V\left(x_{1}, \ldots, x_{n-1}, x_{0}, x_{n+2}\right)} .
\end{aligned}
$$

Since $x_{i}, x_{n+2} \in E \cup E_{1}$, the values $f\left(x_{i}\right), f\left(x_{n+2}\right)$ vary in a bounded set. Therefore by an argument similar to that used for $x_{n+1}=x_{0}$ and $x_{n+2}=x$ we infer the existence of a positive number $\delta_{1}^{\prime}$ such that $f(x)-f\left(x_{0}\right)<\varepsilon$ holds for $x \epsilon\left(x_{0}, x_{0}+\delta_{1}^{\prime}\right) \frown E_{0}^{\prime \prime}$. By the same method as those we have used lately we find that there exist $\delta_{2}^{\prime}>0$ and a set $E_{0}^{\prime \prime \prime}$ with $m\left[\left(x_{0}-\delta_{2}^{\prime}, x_{0}\right)-\right.$ $\left.-E_{0}^{\prime \prime \prime}\right]=0$ such that $x \epsilon\left(x_{0}-\delta_{2}^{\prime}, x_{0}\right) \cap E_{0}^{\prime \prime \prime}$ implies $f(x)-f\left(x_{0}\right)>-\varepsilon$. Finally, there exist $\delta^{*}>0$ and a set $E_{0}^{*}$ with $m\left[\left(x_{0}-\delta^{*}, x_{0}+\delta^{*}\right)-\right.$ $\left.-E_{0}^{*}\right]=0$ such that $\left|f(x)-f\left(x_{0}\right)\right|<\varepsilon$ holds for $x \in\left(x_{0}-\delta^{*}, x_{0}+\delta^{*}\right) \cap E_{0}^{*}$. In this part of our proof wo have supposed that $m\left(a, x_{0}\right) \cap E>0$. If $m\left(a, x_{0}\right) \cap E=0$ we apply the method used above to the function $g(x)=(-1)^{n+1} f(-x)$ defined over $(-b,-a)$. Thus the point $x_{0}$ can be chosen arbitrarily in $(a, b)$. Applying lemma 2, we conclude that the function $f$ is continuous on $(a, b)$. This completes the proof.

Analogously we can prove

THEOREm 2. If the J-convex function $f$ of the $n$-th order over $(a, b)$ 
is bounded on a set $E C(a, b)$ of the second category which satisfies the Baire condition, then it is continuous on $(a, b)$.

T. Popoviciu has proved theorem 1 in the special case when $E=$ $=(a, b)$ (see [4]). Theorems 1 and 2 for a J-convex function of order 1 over $(a, b)$ have been proved in [3] and [2], respectively.

Now let us give certain immediate conclusions of those theorems:

1) If the function $f$ is measurable and $J$-convex of the $n$-th order on $(a, b)$, then it is continuous in that interval (see [4], and for $n=1$ $[6])$.

2) Let a J-convex function $f$ of the $n$-th order over $(a, b)$ satisfy the Baire condition in $(a, b)$. Then it is continuous over $(a, b)$ (for the case of $n=1$ see [2]).

3 ) Every $J$-convex function $f$ of the $n$-th order over $(a, b)$ is either continuous or discontinuous at each point of $(a, b)$.

4) Let $f$ be bounded on a set $E$ of positive measure (of the second category satisfying the Baire condition) contained in $(a, b)$. Let

$$
\frac{\Delta_{h}^{n} f(x)}{h^{n}} \underset{\langle a, \beta\rangle}{\Rightarrow} g(x)
$$

with $h \rightarrow 0$ for every closed interval $\langle\alpha, \beta\rangle \subset(a, b)$ (the symbol $\overrightarrow{\vec{a}, \beta\rangle}$ denotes uniform convergence over $\langle\alpha, \beta\rangle)$, where $g(x) \geqslant M_{a \beta}$ for $x \in\langle\alpha, \beta\rangle$. Then $g=f^{(n)}$. Moreover, $g$ is continuous over $(a, b)$.

In the case of $E=(a, b)$ this result was earlier proved by Marchaud (see [1]). If $f$ is measurable, compare [7] and [5], p. 52 .

Proof of 4). It is sufficient to prove that $f$ is continuous; then by the Marchaud theorem we obtain our result. We remark that

$$
\frac{\Delta^{n}\left[f(x)+c x^{n}\right]}{h^{n}}=\frac{\Delta_{h}^{n} f(x)}{h^{n}}+c n !_{\langle a, \beta\rangle} g(x)+c n ! .
$$

Since for $e>-M_{\alpha \beta} / n$ ! we have $[g(x)+c n !]>0$, there exists $\delta>0$ such that $0<h<\delta$ implies $\Delta_{h}^{n}\left[f(x)+c x^{n}\right] \geqslant 0$ over $\langle\alpha, \beta\rangle$. According to theorem 1 (2) we obtain the continuity of the function $f$ over $(a, b)$.

\section{References}

[1] A. Marchaud, Sur les dérivées et sur les différences des fonotions de variables réelles, Journal de Mathématiques 6 (1927), p. 337-425.

[2] Z. Ciesielski and W. Orlicz, Some remarks on convergenoe of functionals on bases, Studia Math. 16 (1958), p. 335-352.
[3] A. Ostrowski, Über die Funktionalgleichungen der Exponentialfunktion und verwandte Funktionalgleichungen, Jahresbericht der Deutschen Matematiker. Vereinigung, 38 (1929), p. 54-62.

[4] T. Popoviciu, Sur quelques propriétés des fonotions d'une ou de deux varia. bles réelles, Mathematica 8 (1934), p. 1-85.

[5] - Les fonctions convexes, Paris 1945

[6] W. Sierpiński, Sur les fonotions convexes mesurables, Fundamenta Mathematicae 1(1920), p. 125-129.

[7] H. Whitney, Derivatives, difference quotients and Taylor's formula $I$, Bull. Amer. Math. Soc. 40 (1934), p. 89-94.

Regu par la Rédaction le 5. 10.1957 